\title{
Violencia de Género: Perfil de Mujeres con Ayuda Social
}

\section{Domestic Violence: Women's Profile With Social Care}

\author{
Francisco González Sala y Adelina Gimeno Collado \\ Universidad de Valencia
}

\begin{abstract}
Resumen. Se ofrece el perfil de la mujer que ha sufrido malos tratos, a partir de un estudio con 297 mujeres que reciben ayudas sociales por parte del Ayuntamiento de Valencia. La incidencia del maltrato en este grupo es del $37,3 \%$. El perfil de estas mujeres, en comparación con las mujeres del mismo grupo que no han recibido malos tratos se caracteriza por ser de etnia paya, estructura familiar monoparental, estado civil separada, con varias relaciones de pareja previas y con problemas de salud psicológica. En otras variables que caracterizan a las mujeres receptoras de estas ayudas, tales como nivel de estudios, situación laboral y apoyos familiares y extrafamiliares, no existen diferencias significativas. A partir de estos datos se plantean orientaciones para la prevención e intervención comunitaria.

Palabras clave: perfil de la mujer maltratada, ayudas sociales, prevención, intervención comunitaria.
\end{abstract}

\begin{abstract}
The profile of women having suffered domestic violence is presented. It is based on a study made on 297 women who receive social care from the Valencia Council. 37,3\% of the women in this group has suffered domestic violence. The profile of these women, compared with the ones belonging to the same group who don't suffer domestic violence, is characterized by the following features: non-gypsy ethnic group, one-parent familiar structure, marital status separated, several previous sentimental relationships, and psychological problems. In other features which characterize the women receiving social care, like studies level, labour situation, familiar and non-familiar support, no significant differences where appreciated. Based on the present information's, advise on prevention and community intervention is considered.

Key words: profile of women having suffered domestic violence, social care, prevention, community intervention.
\end{abstract}

\section{Justificación}

Según diferentes estudios realizados por el Consejo de Europa, una de cada cuatro mujeres europeas experimentará la violencia de género alguna vez en su vida (Koop CE, 1989). Datos del Ministerio de Asuntos

La correspondencia sobre este artículo puede dirigirse al primer autor a la Universidad de Valencia. Facultad de Psicología. Dep. Psicología Evolutiva. Av. Blasco Ibáñez, 21. 46019 Valencia, o a través del correo electrónico: francisco.gonzalez-sala@uv.es
Sociales (2002) hablan que un 4\% de la población adulta femenina sufre malos tratos, aunque las estadísticas sostienen que el 16,5\% de las mujeres ha manifestado haber sufrido maltrato en algún momento de su vida (Echeburua, 2004). Fontanil, Ezama, Fernández, Gil, Herrero y Paz (2005) sitúan en un $20 \%$ las cifras del maltrato a la mujer en la pareja.

Pero estos datos, acerca de la prevalencia de los malos tratos, sólo reflejan una parte del problema, lo que se ha venido en llamar la punta del iceberg. El 
silencio de las propias mujeres y del entorno social próximo, reflejan una realidad que estremece. En este sentido, estudios vigentes indican que la mujer permanece en la relación violenta una media de diez años antes de denunciar (Echeburua, Sarausúa, Zubizarreta, del Corral, 1990). Abril (1999), Benítez (1998), UNICEF (2000), Watts y Zimmerman (2002) apuntan las razones que contribuyen a reducir la probabilidad de denunciar: el miedo a continuar o reanudar la convivencia con el maltratador después de la denuncia, la falta de confianza en el sistema judicial, la dependencia económica, la dependencia afectiva, la resitencia a aceptar el fracaso en la relación, los sentimientos de culpa y verguienza, la consideración de que el problema pertenece al ámbito privado, entre otras. Corral (2000) resalta la importancia de la dependencia emocional, del aislamiento social y de la dependencia económica de la mujer. Strube y Barbour (1984) el compromiso matrimonial a aceptar y someterse al marido, la dependencia económica, la falta de empleo y el no tener donde ir. Otros estudios indican que el bajo nivel cultural, -ya que aproximadamente la mitad tiene estudios primarios- y la baja preparación laboral, especialización laboral - labores domésticas la ocupación principal- como causas que determinan que una mujer permanezca en la relación de violencia (Departament de Benestar Social, 2001).

Según Echeburúa, Corral, Sarasua y Zubizarreta (1996), el perfil de las mujeres maltratadas en los centros asistenciales es el de una mujer con un nivel cultural bajo, con trabajos poco cualificados y desempleo, dependiente económicamente del marido, con un escaso apoyo social, con cargas familiares importantes y hacinamiento.

Si bien, son muchos los estudios centrados en el perfil de la mujer maltratada o del agresor, esto supone una visión reduccionista del problema, sobre todo tras la detección de casos, a menudo más silenciados, en clases culturales y económicamente más altas, por lo que se plantea la necesidad de estudiar el problema de la violencia de género desde una perspectiva más amplia, que tenga en cuenta otros factores de carácter más estructural, así el modelo ecológico aplicado al campo de la violencia familiar, apunta como factores de riesgo dentro del ecosistema - el estrés económico, el desempleo, el aislamiento social y el alcoholismo , aspectos algunos de ellos que se recogen en el presente estudio. Desde esta perspectiva se apuntan como factores asociados a la violencia, la vulnerabili- dad social de la mujer, la desigualdad entre hombres y mujeres en los valores sociales y en los códigos jurídicos, así como en los recursos y en el poder (Cabruja, 2004; Fernández Villanueva, 2004).

La exclusiva elaboración de perfiles de mujeres maltratadas puede ayudar a elaborar programas de prevención e intervención de la violencia de género, pero como recogen Neiding y Friedman (1988) pueden ser poco adecuados por estigmatizantes, en tanto que el resultado de la violencia obedece más a múltiples factores que a factores individuales. Giddens (2000) y Trujado, Nava, Tejada y Gutiérrez (2006) con vistas a prevenir las conductas violentas en el futuro hablan de abordar esta problemática desde un enfoque global acerca del origen y mantenimiento de la violencia.

El presente trabajo tiene como objetivo analizar la incidencia de la violencia machista en población usuaria de Servicios Sociales, describir el perfil de las mujeres víctimas de la violencia, y comparar, la muestra de mujeres que ha padecido malos tratos con la muestra que no los ha sufrido, todo ello con vistas a conocer la situación real de la mujer maltratada que recibe ayuda social, con el fin de orientar la intervención y proponer medidas eficaces de prevención y actuación en mujeres maltratadas de clase social baja. El presente enfoque no excluye la existencia de malos tratos en otros grupos sociales, simplemente se centra en la población que por la carencia de recursos resulta más vulnerable incrementando el riesgo de una exclusión social irreversible.

\section{Metodología}

\section{Descripción de la muestra}

La muestra está constituida por familias que reciben ayudas sociales de tipo P.E.R. (Prestaciones Económicas Regladas) y P.E.P. (Prestaciones Económicas por Protección) del Ayuntamiento de Valencia. Son un total de 294 mujeres con edades entre los 18 y los 59 años.

\section{Instrumento y variables seleccionadas}

El instrumento empleado para recoger la información ha sido "Registro y valoración de familias procedentes de Servicios Sociales" utilizado para ela- 
borar los expedientes de los casos atendidos en las diferentes sedes de Servicios Sociales Municipales. La información que contenían dichos expedientes fue obtenida por los propios técnicos municipales mediante varias entrevistas personales con las mujeres beneficiarias de las ayudas sociales.

La información existente se ha organizado en las dimensiones seleccionadas, que obedecen a una revisión de la bibliografía de familias procedentes de Servicios Sociales y mujeres maltratadas. En esta organización se utilizó el criterio de consenso de jueces, que eran tres especialistas en el tema, dos psicólogos y un trabajador social. Estas dimensiones han sido: Características socioculturales y familiares, que incluye los siguientes indicadores - Etnia, Nivel cultural, Estado civil, Relaciones de pareja anteriores, Estructura familiar y Cargas familiares -, Nivel laboral - Situación laboral y Actividad laboral, la dimensión Red social de apoyo cuyos indicadores son - Apoyo social extrafamiliar: amigos y vecinos y Apoyo de la familia extensa -, en cuanto a la dimensión Salud los indicadores son - Salud psicológica y Asistencia psicológica.

\section{Análisis efectuados}

Mediante el paquete estadístico SPSS 15.0, hemos realizado tablas de contingencia, que nos permite conocer la incidencia y el perfil de las víctimas de violencia de género a partir de las dimensiones de estudio. Cogeremos las frecuencias y porcentajes de casos incluidos en cada dimensión.

Para la comparación de los perfiles de mujeres que han sufrido malos tratos y aquéllas que no los han sufrido, en cada uno de los indicadores objeto de estudio y buscar diferencias entre perfiles, realizaremos pruebas Chi cuadrado, utilizando como variable agrupadora la presencia de malos tratos.

En los casos que no se cumplía la condición para aplicar este tipo de prueba, es decir, aquéllos donde la frecuencia esperada fuera menor del $20 \%$, aplica- remos la prueba Chi cuadrado corregida por Monte Carlo y el estadístico exacto de Fisher, siempre empleando un intervalo de confianza del $95 \%$.

\section{Resultados}

A continuación se presentan los resultados en dos apartados: Incidencia de los malos tratos, y Descripción y análisis comparativo del perfil de la mujer maltratada diferenciando cuatro ámbitos: personal, familiar, laborar y red social de apoyo, incluyendo en cada ámbito los indicadores o dimensiones seleccionadas para configurar el perfil. En el ámbito personal incluímos etnia, nivel cultural y estado civil, en el ámbito familiar relaciones de pareja anteriores, estructura familiar y cargas familiares, en el ámbito laboral situación laboral y actividad laboral actual y en la red social de apoyo incluimos red extrafamiliar y familia extensa.

\section{Incidencia de los malos tratos}

La incidencia de la violencia de género en la muestra de mujeres que recibían ayudas sociales es del $37,3 \%$.

\section{Descripción y análisis del perfil de mujer maltratada}

\section{Características socioculturales personales}

a) Etnia.

Este indicador está compuesto por cuatro categorías -Payos, Gitanos, Inmigrantes y Mixto-, categoría esta última formada por parejas de diferente grupo étnico. Según este indicador, entre las mujeres que han sufrido malos tratos, el más representado es el de mujeres payas, en concreto un $58,3 \%$. El resto de datos se pueden consultar en la tabla $n^{\circ} .2$.

Tabla 1. Incidencia de la violencia de género

\begin{tabular}{lccc}
\hline & SÍ & NO & TOTAL \\
\hline TOTAL & 109 & 183 & 292 \\
\hline Porcentaje & $\mathbf{3 7 , 3}$ & $\mathbf{6 2 , 7}$ & 100 \\
\hline
\end{tabular}


Tabla 2. Etnia

\begin{tabular}{|c|c|c|c|c|c|c|}
\hline \multirow[b]{2}{*}{ M } & & \multicolumn{4}{|c|}{ ETNIA } & \multirow[t]{2}{*}{ TOTAL } \\
\hline & & Payos & Gitanos & Inmigrantes & Mixto & \\
\hline $\mathbf{L}$ & SI & 63 & 20 & 17 & 8 & 108 \\
\hline $\mathbf{T}$ & Porcentaje & 58,3 & 18,5 & 15,7 & 7,4 & 100 \\
\hline $\mathbf{R}$ & NO & 71 & 64 & 39 & 8 & 182 \\
\hline A & Porcentaje & 39,0 & 35,2 & 21,4 & 4,4 & 100 \\
\hline 0 & TOTAL & 134 & 84 & 56 & 16 & 290 \\
\hline
\end{tabular}

Al comparar los casos existentes en cada grupo étnico, obtenemos en la prueba Chi cuadrado un valor de 14,211, con $3 \mathrm{gl}$. y un nivel de significación de 0.003 , por lo que podemos decir que el grupo étnico sí que guarda relación con el maltrato. Observamos que predomina significativamente el grupo de payos siendo el grupo de gitanos y el de inmigrantes mucho menor.

b) Nivel cultural.

Este indicador está compuesto por cinco categorías -Sin estudios, EGB sin finalizar, Graduado, Medios y Superiores-. En la tabla $n^{\circ} 3$ observamos que las categoría con mayor porcentaje de casos son las de más bajo nivel, el 63,9\% sin estudios y con EGB sin finalizar.

La prueba Chi cuadrado aplicada da un valor de 5,822, corregida por el estadístico exacto de Fisher con un valor de 5,386, para $4 \mathrm{gl}$. le corresponde un nivel de significación de 0.234 , tras la corrección de Monte Carlo, que nos ofrece una mejor estimación. Lo que indica que el nivel cultural es independiente del maltrato. c) Estado civil.

Este indicador está compuesto por cinco categorías -Casadas, Solteras que no conviven con pareja, Viudas, Separadas y Convivencia en pareja-. Según los datos, ver tabla $\mathrm{n}^{\circ} .4$, el maltrato predomina entre las mujeres separadas y solteras, que constituyen en su conjunto un $82,5 \%$.

Según la prueba Chi cuadrado realizada para relacionar ambas variables, obtenemos un valor de Chi cuadrado de 23,073, corregida por el estadístico exacto de Fisher con un valor de 22,625, para 4 gl. le corresponde un nivel de significación de 0,000 , tras la corrección de Monte Carlo, podemos decir que el maltrato no es independiente del estado civil de la mujer, sino que predominan las mujeres separadas.

\section{Características familiares}

a) Relaciones de pareja anteriores.

Este indicador está compuesto por dos categorías -Sí ha habido otras relaciones y No ha habido otras relaciones-. Según los datos, el 49,5\% de las muje-

\begin{tabular}{|c|c|c|c|c|c|c|c|}
\hline \multicolumn{8}{|c|}{ Tabla 3. Nivel cultural } \\
\hline & & \multicolumn{5}{|c|}{ NIVEL CULTURAL } & \multirow[t]{2}{*}{ TOTAL } \\
\hline $\mathbf{M}$ & & Sin estudios & E.G.B. sin finalizar & Graduado & Medios & Superior & \\
\hline $\mathbf{L}$ & SI & 37 & 32 & 24 & 15 & 0 & 108 \\
\hline $\mathbf{T}$ & Porcentaje & 34,3 & 29,6 & 22,2 & 13,9 & $\mathbf{0}$ & 100 \\
\hline $\mathbf{R}$ & NO & 74 & 50 & 41 & 14 & 4 & 183 \\
\hline $\mathbf{A}$ & Porcentaje & 40,4 & 27,3 & 22,4 & 7,7 & 2,2 & 100 \\
\hline $\mathbf{0}$ & TOTAL & 111 & 82 & 65 & 29 & 4 & 291 \\
\hline
\end{tabular}


Tabla 4. Estado civil

\begin{tabular}{|c|c|c|c|c|c|c|c|}
\hline \multirow[b]{2}{*}{ M } & & \multicolumn{5}{|c|}{ ESTADO CIVIL } & \multirow[t]{2}{*}{ TOTAL } \\
\hline & & Casada & Soltera & Viuda & Separada & Conviv. & \\
\hline $\mathbf{L}$ & SI & 17 & 37 & 1 & 53 & 1 & 109 \\
\hline $\mathrm{T}$ & Porcentaje & 15,6 & 33,9 & 0,7 & 48,6 & 0,9 & 100 \\
\hline $\mathbf{R}$ & NO & 50 & 69 & 11 & 45 & 8 & 183 \\
\hline A & Porcentaje & 27,3 & 37,7 & 6,0 & 24,6 & 4,4 & 100 \\
\hline O & TOTAL & 67 & 106 & 12 & 98 & 9 & 292 \\
\hline
\end{tabular}

res que han sufrido malos tratos no han tenido otras relaciones (tabla $\mathrm{n}^{\circ} .5$ ), es decir, los han padecido en su primera relación de pareja estable que tuvieron.

Al comparar la variable maltrato con esta nueva variable mediante la prueba Chi cuadrado, obtenemos un valor de 7,819, al que para $1 \mathrm{gl}$. corresponde un nivel de significación de 0,005 . Se trata pues de un nivel de significación que indica la relación entre maltrato y la existencia de relaciones anteriores.

Interpretamos que el mayor porcentaje de mujeres no maltratadas, no han tenido ninguna relación anterior. Pero hay que tener en cuenta que según estos mismos datos, entre las mujeres con relaciones de pareja anteriores son menos los casos de maltrato (55 frente a 62), lo que nos lleva a interpretar con cautela los resultados. Si analizamos la distribución de datos por columnas, vemos que los casos de no maltrato casi duplican al de maltratadas cuando se trata de la primera pareja, mientras que cuando hubo otras relaciones anteriores casi la mitad de los casos (55 casos) fueron víctimas de maltrato.

b) Estructura familiar.

Este indicador está compuesto por cuatro catego- rías -Monoparental, Nuclear completa, Reconstituida y Extensa-. Atendiendo al tipo de estructura familiar, predomina un tipo de estructura monoparental, tanto entre las mujeres que han sufrido malos tratos, un $69,7 \%$, como entre las que no los han padecido, un $47,5 \%$ de los casos (tabla $n^{\circ} .6$ ).

Podemos decir que existen diferencias estadísticamente significativas en cuanto a la presencia de malos tratos y la estructura familiar, si tenemos en cuenta los datos encontrados en el análisis efectuado al respecto, siendo el valor de Chi cuadrado de 20,609 , al que para $3 \mathrm{gl}$. le corresponde un nivel de significación de 0,000 . Son las familias monoparentales las que incluyen mayor porcentaje de mujeres que han sufrido maltrato, y si nos fijamos sólo en las monoparentales vemos que lo han sufrido casi la mitad de los 163 casos de esta categoría. En cambio si nos fijamos en las nucleares completas, la proporción a favor del no maltrato es muy alta y superior que en el resto de estructuras familiares.

c) Cargas familiares.

Para este indicador hemos considerado el número de hijos por familia, diferenciando cinco categorías

Tabla 5. Relaciones de pareja anteriores

\begin{tabular}{c|cccc}
\hline \multicolumn{2}{c}{ RELACIONES PAREJA ANTERIORES } & TOTAL \\
\hline $\mathbf{M}$ & & Si otras relaciones & No otras relaciones \\
$\mathbf{A}$ & & 55 & 54 & 109 \\
$\mathbf{L}$ & SI & $\mathbf{5 0 , 5}$ & $\mathbf{4 9 , 5}$ & 100 \\
$\mathbf{T}$ & Porcentaje & 62 & 121 & 183 \\
$\mathbf{R}$ & NO & $\mathbf{3 3 , 9}$ & $\mathbf{6 6 , 1}$ & 100 \\
$\mathbf{A}$ & Porcentaje & $\mathbf{1 1 7}$ & $\mathbf{1 7 5}$ & 292 \\
$\mathbf{T}$ & TOTAL & & & \multirow{2}{*}{} \\
$\mathbf{n}$ & & &
\end{tabular}


Tabla 6. Estructura familiar

\begin{tabular}{|c|c|c|c|c|c|c|}
\hline \multirow[b]{2}{*}{ M } & & \multicolumn{4}{|c|}{ ESTRUCTURA FAMILIAR } & \multirow[t]{2}{*}{ TOTAL } \\
\hline & & Monoparent. & Nuclear & Extensa & Conviven. & \\
\hline $\mathbf{L}$ & SI & 76 & 14 & 10 & 9 & 109 \\
\hline$T$ & Porcentaje & 69,7 & 12,8 & 9,2 & 8,3 & 100 \\
\hline $\mathbf{R}$ & NO & 87 & 54 & 9 & 33 & 183 \\
\hline A & Porcentaje & 47,5 & 29,5 & 4,9 & 18,0 & 100 \\
\hline $\mathbf{0}$ & TOTAL & 163 & 68 & 19 & 42 & 292 \\
\hline
\end{tabular}

-Un menor, dos menores, tres menores, cuatro menores y cinco o más menores-. Según los datos, el $65,1 \%$ de las mujeres maltratadas tiene entre uno y dos menores a su cargo. Los porcentajes para cada una de las categorías quedan recogidos en la tabla $n^{\circ} .7$.

Por otra parte, podemos decir que no existen diferencias estadísticamente significativas a la hora de haber sufrido o no malos tratos en función de las cargas familiares, según los datos obtenidos en la comparación, siendo el valor para Chi cuadrado de 5,861, 4 gl. y un nivel de significación de 0,581. interpretable, teniendo en cuenta que en las tres categorías estamos hablando de un nivel de ingresos muy bajo, tanto que hace necesaria la concesión de ayudas sociales. Veamos además, lo que entendemos por trabajo a partir de las aclaraciones que hacemos en el apartado siguiente "Actividad laboral", pues se trata de actividades muy precarias, aunque sean una fuente de ingresos.

b) Actividad laboral.

Diferenciamos ocho categorías en función del

Tabla 7. Cargas familiares

CARGAS FAMILIARES ( $\mathbf{N}^{\circ}$. de menores)

TOTAL

\begin{tabular}{c|ccccccc}
\hline $\mathbf{M}$ & & Uno & Dos & Tres & Cuatro & \multicolumn{2}{c}{ Cinco o más } \\
$\mathbf{A}$ & & 37 & 34 & 24 & 9 & 5 & 109 \\
$\mathbf{L}$ & SI & $\mathbf{3 3 , 9}$ & $\mathbf{3 1 , 2}$ & $\mathbf{2 2 , 0}$ & $\mathbf{8 , 3}$ & $\mathbf{4 , 6}$ & 100 \\
$\mathbf{T}$ & Porcentaje & 69 & 57 & 27 & 17 & 12 & 183 \\
$\mathbf{R}$ & NO & $\mathbf{3 7 , 9}$ & $\mathbf{3 1 , 3}$ & $\mathbf{1 4 , 8}$ & $\mathbf{9 , 3}$ & $\mathbf{6 , 6}$ & 100 \\
$\mathbf{A}$ & Porcentaje & $\mathbf{1 0 6}$ & $\mathbf{9 1}$ & $\mathbf{5 1}$ & $\mathbf{2 6}$ & $\mathbf{1 7}$ & 292 \\
$\mathbf{T}$ & TOTAL & & & & & \\
$\mathbf{O}$ & & & & &
\end{tabular}

\section{Ámbito laboral}

a) Situación laboral.

Este indicador presenta tres categorías -Trabajo, Desempleo y Pensionista-. Atendiendo a la misma, podemos decir que entre las mujeres maltratadas predomina el desempleo en un $81,7 \%$ de los casos (tabla $\mathrm{n}^{\circ} .8$ ).

La relación entre esta variable y el maltrato, muestra que no existe relación entre ambas. Ya que hemos encontrado un valor de Chi cuadrado de 3,613 , al que para $2 \mathrm{gl}$. corresponde un nivel de significación de 0,164 . Este es un resultado fácilmente tipo de actividad laboral que detallamos al pié de la tabla $n^{\circ}$. 9. En ella observamos que la actividad laboral más frecuente (el 62,3\%), corresponde a actividades relacionadas con los servicios domésticos y de limpieza.

Al comparar ambas variables, maltrato y actividad laboral, encontramos tras aplicar la prueba Chi cuadrado, que existen diferencias estadísticamente significativas, concluyendo que ambas variables están relacionadas. El valor de Chi cuadrado es de 15,596, el estadístico de Fisher con un valor de 15,308, al que para $7 \mathrm{gl}$. le corresponde un nivel de significación, que corregido por Monte Carlo, es de 0,024. 
Tabla 8. Situación laboral

SITUACIÓN LABORAL

TOTAL

\begin{tabular}{l|ccccc}
\hline $\mathbf{M}$ & & Trabajo & Desempleo & Pensionista \\
$\mathbf{A}$ & & 10 & 89 & 10 & $\mathbf{9 , 2}$ \\
$\mathbf{L}$ & SI & $\mathbf{9 , 2}$ & $\mathbf{8 1 , 7}$ & 8 & 109 \\
$\mathbf{T}$ & Porcentaje & 12 & 163 & $\mathbf{4 , 4}$ & 183 \\
$\mathbf{n y y y y y} \mathbf{R}$ & NO & $\mathbf{6 , 6}$ & $\mathbf{8 9 , 1}$ & $\mathbf{1 8}$ & 100 \\
$\mathbf{A}$ & Porcentaje & $\mathbf{2 2}$ & $\mathbf{2 5 2}$ & 292 \\
$\mathbf{T}$ & TOTAL & & & \\
$\mathbf{O}$ & & &
\end{tabular}

En esta comparación llama la atención que son las mujeres con trabajos menos cualificados y que podemos catalogar como actividades marginales, si tenemos en cuenta los grupos de mendicidad y recogida de chatarra y el de venta no reglada, las que menos malos tratos reciben, mientras que son las mujeres con trabajos más cualificados, casos de servicios domésticos y sector servicios, las que más malos tratos manifiestan haber recibido.
Podemos decir que no existen diferencias estadísticamente significativas a la hora de haber sufrido o no malos tratos en función del apoyo extrafamiliar, según los datos obtenidos en la comparación, siendo el valor para Chi cuadrado de 1,433, 4 gl. y un nivel de significación de 0,849 . Observamos que las categorías inexistente y ocasional presentan los porcentajes más altos en ambos casos; como tendencia de respuesta vemos que en las categorías apoyo fre-

\begin{tabular}{|c|c|c|c|c|c|c|c|c|c|c|}
\hline \multicolumn{11}{|c|}{ Tabla 9. Actividad laboral } \\
\hline & & \multicolumn{8}{|c|}{ ACTIVIDAD LABORAL } & \multirow[t]{2}{*}{ TOTAL } \\
\hline $\mathbf{M}$ & & G1 & $\mathrm{G} 2$ & G3 & G4 & G5 & G6 & $\mathrm{G} 7$ & G8 & \\
\hline \multirow{2}{*}{$\begin{array}{l}\mathbf{A} \\
\mathbf{L} \\
\mathbf{T}\end{array}$} & SI & 1 & 11 & 1 & 66 & 16 & 0 & 1 & 10 & 106 \\
\hline & Porcentaje & 0,9 & 10,4 & 0,9 & 62,3 & 15,1 & $\mathbf{0}$ & 0,9 & 9,4 & 100 \\
\hline \multirow{3}{*}{$\begin{array}{c}\mathbf{R} \\
\mathbf{A} \\
\mathbf{T} \\
\mathbf{O}\end{array}$} & $\mathrm{NO}$ & 13 & 25 & 5 & 89 & 18 & 5 & 0 & 22 & 177 \\
\hline & Porcentaje & 7,3 & 14,1 & 2,8 & 50,3 & 10,2 & 2,8 & $\mathbf{0 , 0}$ & 12,4 & 100 \\
\hline & TOTAL & 14 & 36 & 6 & 155 & 32 & 5 & 1 & 32 & 283 \\
\hline \multicolumn{11}{|c|}{$\begin{array}{l}\text { Grupo 3. Venta ambulante reglada. Grupo 4. Cuidado de ancianos. Servicios domésticos. Limpieza } \\
\text { Grupo 5. Camarera, cocinera, auxiliar admón., dependienta, encuestadora, vigilante, conserje.. } \\
\text { Grupo 6. Albañil, peón, pintor, mecánico, electricista, campo, carga y descarga, pulidor, transportista } \\
\text { Grupo } 7 \text { OTRAS. Prof. Universidad, soldado profesional. Grupo } 8 \text {. Amas de casa y pensionistas }\end{array}$} \\
\hline
\end{tabular}

\section{Red social de apoyo}

a) Apoyo social extrafamiliar: amigos y vecinos. Este indicador está compuesto por cinco categorías -Inexistentes, esporádicos, variables, frecuentes y siempre-. Según los datos, estas mujeres se caracterizan por carecer de apoyos por parte de amigos y vecinos, ya que el $45 \%$ no presenta apoyos en este sentido, mientras que es la categoría que incluye apoyos de modo continuo, la que presenta el porcentaje más bajo (tabla $n^{\circ}$. 10). cuente y siempre hay porcentajes superiores en el grupo de maltratadas, mientras es superior el porcentaje de casos en las categorías inexistente y ocasional en los casos sin malos tratos.

b) Apoyos de la familia extensa.

El indicador "apoyos de la familia extensa" está compuesto por cinco categorías -Inexistentes, esporádicos, variables, frecuentes y siempre-. La distribución de los casos se encuentra en la tabla $n^{\circ} .11$, en ella vemos que entre las mujeres que han sufrido 
Tabla 10. Apoyo social extrafamiliar. amigos y vecinos

\begin{tabular}{|c|c|c|c|c|c|c|c|}
\hline \multirow[b]{2}{*}{ M } & & \multicolumn{5}{|c|}{ APOYO SOCIAL EXTRAFAMILIAR } & \multirow[t]{2}{*}{ TOTAI } \\
\hline & & Inexist. & Ocasional & Variable & Frecuente & Siempre & \\
\hline $\mathrm{L}$ & SI & 49 & 15 & 15 & 25 & 5 & 109 \\
\hline $\mathrm{T}$ & Porcentaje & 45,0 & 13,8 & 13,8 & 22,9 & 4,6 & 100 \\
\hline $\mathbf{R}$ & NO & 88 & 28 & 27 & 33 & 6 & 182 \\
\hline A & Porcentaje & 48,4 & 15,4 & 14,8 & 18,1 & 3,3 & 100 \\
\hline $\mathbf{O}$ & TOTAL & 137 & 43 & 42 & 58 & 11 & 291 \\
\hline
\end{tabular}

malos tratos, el 30,3\% no cuenta con ningún tipo de apoyo por parte de su familia extensa.

Al comparar las variables maltrato y apoyos de la familia extensa mediante la prueba Chi cuadrado, encontramos un valor de 0,966 , para $4 \mathrm{gl}$. al que corresponde un nivel de significación de 0,915, podemos decir que no existe relación estadísticamente significativa entre ambas variables. mujeres que han padecido una situación de maltrato, al tiempo que la buena salud corresponde en mayor porcentaje a las mujeres sin experiencias del maltrato $(\mathrm{el} 67,2 \%)$.

b) Asistencia psicológica.

El indicador "asistencia psicológica" está compuesto por dos categorías -Asisten a salud mental y

\begin{tabular}{|c|c|c|c|c|c|c|c|}
\hline \multirow[b]{2}{*}{ M } & & \multicolumn{5}{|c|}{ APOYOS DE LA FAMILIA EXTENSA } & \multirow[t]{2}{*}{ TOTAL } \\
\hline & & Inexist. & Ocasional & Variable & Frecuente & Siempre & \\
\hline L & $\begin{array}{c}\text { SI } \\
\text { Porcentaje }\end{array}$ & $\begin{array}{c}33 \\
\mathbf{3 0 , 3}\end{array}$ & $\begin{array}{c}19 \\
17,4\end{array}$ & $\begin{array}{c}19 \\
17,4\end{array}$ & $\begin{array}{c}22 \\
\mathbf{2 0 , 2}\end{array}$ & $\begin{array}{c}16 \\
14,7\end{array}$ & $\begin{array}{l}109 \\
100\end{array}$ \\
\hline $\begin{array}{l}\mathbf{R} \\
\mathbf{A}\end{array}$ & $\begin{array}{c}\text { NO } \\
\text { Porcentaje }\end{array}$ & $\begin{array}{c}49 \\
26,8\end{array}$ & $\begin{array}{c}31 \\
16.9\end{array}$ & $\begin{array}{c}29 \\
15,8\end{array}$ & $\begin{array}{c}43 \\
23.5\end{array}$ & $\begin{array}{c}31 \\
169\end{array}$ & $\begin{array}{l}183 \\
100\end{array}$ \\
\hline $\begin{array}{l}\mathrm{T} \\
\mathrm{O}\end{array}$ & TOTAL & 82 & 50 & 48 & 65 & 47 & 292 \\
\hline
\end{tabular}

\section{Salud}

\section{a) Salud psicológica.}

En esta variable diferenciamos tres categorías -Mala, Con altibajos y Buena salud- (tabla $\left.\mathrm{n}^{\mathrm{o}} 12\right)$. En ella vemos que un $23 \%$ de las mujeres presenta problemas de salud.

Además, podemos decir que existe una relación estadísticamente significativa entre la presencia de malos tratos y la variable salud psicológica; si tenemos en cuenta los resultados de la prueba Chi cuadrado, en la que se obtiene un valor de 15,127 , para el que con 2 gl. corresponde un nivel de significación de 0,001 . Aspecto que puede ser explicado por los mayores problemas emocionales que sufren las
No asisten a salud mental-. Según los datos, entre las mujeres que han sufrido malos tratos, podemos destacar que solamente un $15,7 \%$ ha recibido intervención psicológica (tabla $n^{\circ} .13$ ), de las cuales, en tres casos, dicha intervención provenía de un centro especializado en violencia de género.

En el análisis de datos obtenemos un valor de Chi cuadrado de 0,001 , al que para $1 \mathrm{gl}$. corresponde un nivel de significación de 0,973 , por lo que podemos decir que no existen diferencias estadísticamente significativas entre ambas variables. En este caso los resultados despiertan nuestra preocupación por la falta de asistencia en ese $84,3 \%$ de mujeres maltratadas, y despierta también nuestro interés por conocer las causas por las que no han buscado o conseguido asistencia. 


\begin{tabular}{|c|c|c|c|c|c|}
\hline \multicolumn{6}{|c|}{ Tabla 12. Salud psicológica } \\
\hline & & \multicolumn{3}{|c|}{ SALUD PSICOLÓGICA } & \multirow[t]{2}{*}{ TOTAI } \\
\hline M & & Mala & Con altibajos & Buena & \\
\hline $\mathbf{L}$ & SI & 25 & 36 & 48 & 109 \\
\hline $\mathbf{T}$ & Porcentaje & 22,9 & 33,0 & 44,0 & 100 \\
\hline $\mathbf{R}$ & NO & 25 & 35 & 123 & 183 \\
\hline A & Porcentaje & 13,7 & 19,1 & 67,2 & 100 \\
\hline o & TOTAL & 50 & 71 & 171 & 292 \\
\hline
\end{tabular}

\begin{tabular}{|c|c|c|c|c|}
\hline \multicolumn{5}{|c|}{ Tabla 13. Asistencia psicológica } \\
\hline & & \multicolumn{2}{|c|}{ ASISTENCIA PSICOLÓGICA } & \multirow[t]{2}{*}{ TOTAL } \\
\hline $\mathbf{M}$ & & Asisten a salud mental & No asisten a salud mental & \\
\hline $\mathbf{L}$ & SI & 17 & 91 & 108 \\
\hline $\mathbf{T}$ & Porcentaje & 15,7 & 84,3 & 100 \\
\hline $\mathbf{R}$ & NO & 29 & 157 & 186 \\
\hline A & Porcentaje & 15,6 & 84,4 & 100 \\
\hline O & TOTAL & 46 & 248 & 294 \\
\hline
\end{tabular}

\section{Conclusiones}

Según los resultados expuestos en el apartado anterior, hemos de destacar el elevado porcentaje de mujeres que reconoce haber sufrido malos tratos, en concreto el $37,3 \%$, porcentaje que esperamos sea mayor si tenemos en cuenta otras razones que hacen que se mantenga encubierto este tipo de situaciones, como las creencias culturales, el miedo, la dependencia económica y emocional, las cargas familiares (Corral, 2000; Strube y Barbour, 1984). Por lo que podemos decir que la incidencia de malos tratos en mujeres que reciben ayudas sociales, procedentes de familias multiproblemáticas, está por encima de la incidencia de estudios dirigidos a la población general, (Koop CE, 1989, Echeburua, 2004).

A la hora de interpretar el nivel de incidencia, puede llamar la atención el porcentaje tan alto que ha reconocido sufrir malos tratos, pero hemos de tener en cuenta que en este tipo de población puede resultar más fácil detectar estas situaciones, en la medida que acuden a los Servicios Sociales solici- tando una ayuda de tipo económico para atender las necesidades económicas que presentan. Además, sería interesante conocer el porcentaje de las mujeres de la muestra que ha presentado denuncia, ya que un $80 \%$ de las mujeres no inician trámite judicial alguno (Instituto de la Mujer, 2004); no obstante, el porcentaje significativo en las categorías separadas o solteras indica que con independencia de haber presentado denuncia o no han optado por no convivir con el maltratador.

En relación con el perfil de mujer maltratada con ayudas sociales, decir que es el de una mujer paya, con un nivel cultural muy bajo, al no tener estudios primarios, separada, que forma una familia monoparental con uno o dos menores a su cargo. Los datos avalan la hipótesis de feninización de la pobreza (Zacarés, 2005).

Destacar que prácticamente la mitad de ellas no ha tenido otras relaciones de pareja, de lo cual se deduce que en su primera relación han padecido este tipo de violencia, ello nos lleva a plantearnos la necesidad de programas de prevención de la violencia doméstica a través de la formación, dirigidos a 
población adolescente, con escasos recursos y procedentes de ambientes marginales o deprivados.

Son mujeres con un bajo nivel laboral, caracterizado por el desempleo y por actividades poco cualificadas. Con una red social de apoyo muy deficitaria, tanto a nivel extrafamiliar como por parte de la familia extensa, llegando a hablar de aislamiento social en un porcentaje muy a tener en cuenta, aspectos también señalados por Echeburúa, Corral, Zubizarreta, y Sarasua (1996) en un estudio con mujeres maltratadas que provenían de centros asistenciales. Por lo que respecta a la red social de apoyo y a la familia extensa sería interesante realizar estudios longitudinales que vayan más allá de la instantánea que suponen estos datos, lo que nos daría información sobre la existencia de los apoyos antes y después del maltrato y sobre los apoyos concretos y los beneficios que aportan en relación con las familias que no reciben apoyos.

Son mujeres que presentan problemas de salud psicológica, aunque los datos sólo nos hablen de casi una cuarta parte. Que no reciben ayuda psicológica, y que en muy pocas ocasiones acuden a centros especializados de atención a mujeres víctimas de malos tratos. Ello nos hace plantearnos en qué medida las campañas de difusión de recursos especializados de atención a la mujer son efectivos en este tipo de población, así como la accesibilidad a los mismos.

En cuanto a la comparación de la muestra de mujeres que ha sufrido malos tratos con respecto a la que no los ha padecido, podemos decir que existen diferencias entre ambas en los siguientes indicadores -etnia, estado civil, otras relaciones de pareja, estructura familiar, actividad laboral y salud psicológica-. Ello nos lleva a plantear las siguientes reflexiones, podemos esperar que las mujeres que han sufrido malos tratos se caractericen en mayor medida por ser mujeres separadas y solteras, frente a mujeres que no los han sufrido, caracterizadas en mayor medida por estar casadas, por presentar una estructura monoparental frente a una estructura nuclear o de convivencia, pero la cuestión es, qué papel juega la etnia. Es decir, son los hombres payos los que más maltratan a las mujeres frente a otros grupos, gitanos o inmigrantes, o son las mujeres payas las que reconocen en mayor medida el haber sufrido malos tratos frente a otros grupos étnicos o culturales. En este aspecto, hemos de tener en cuenta otras variables que pueden estar incidiendo en estos resultados, variables culturales, familiares y sociales, que pueden actuar como freno al reconocimiento de esta situación y a la hora de minimizar o justificar una posible agresión.

Entre los indicadores que no existen diferencias estadísticamente significativas podemos mencionar, el nivel cultural, las cargas familiares, los apoyos de amigos y vecinos y de la familia extensa, y la situación laboral, lo cual puede ser esperado, al ser éstas características que definen a las familias multiproblemáticas o procedentes de Servicios Sociales (González y Gimeno, 2006; Consejería de Salud y Bienestar Social de Castilla y León, 1991; Trigo, 1997).

Estos resultados no sólo nos permiten detectar una situación de exclusión social, sino que además nos alertan del riesgo de que la exclusión se perpetúe, debido sobre todo al bajo nivel cultural y laboral de las mujeres, al escaso apoyo familiar y extrafamiliar que reciben y la falta de participación en programas sociales orientados a mejorar su competencia, programas que por diferentes razones que escapan al propósito de este trabajo, no les resultan accesibles.

Teniendo en cuenta la incidencia y el perfil de mujer maltratada con ayudas sociales, procedente de Servicios Sociales, se proponen las siguientes intervenciones:

a) Dirigidas a la atención desde los Centros de Servicios Sociales:

- Crear grupos especializados de atención social y psicológica a mujeres maltratadas, beneficiarias de ayudas sociales, en los propios Centros Municipales.

b) Dirigida a las mujeres:

- Proporcionar atención psicológica y formación con el fin de prevenir que se produzcan nuevas relaciones abusivas.

- Posibilitar la incorporación al mundo laboral, como una forma de luchar contra la feminización de la pobreza, acción que viene recogida en la Ley contra la Violencia de Género aprobada en el 2004.

c) Realizar campañas de difusión de los recursos especializados entre la población marginal o con pocos recursos, así como facilitar la accesibilidad a dichos recursos.

d) Facilitar la accesibilidad a estos recursos, tanto en horario como proximidad geográfica, pero también teniendo los menores que tienen a su cargo. 
Finalmente y aunque resulte obvio, no queremos dejar de insistir en que el apoyo económico es necesario pero insuficiente si no va acompañado de otros programas, de formación y otras ayudas que incremente la formación personal y la preparación laboral de estas mujeres.

\section{Referencias}

Abril, C. (1999). Violencia doméstica: Planteamiento general. En J.D. Martín (coord.), La violencia sobre la mujer en el grupo familiar. Tratamiento jurídico y psicosocial (21-32). Madrid: Codex.

Benítez, M.J. (1998). Violencia intrafamiliar: La mujer maltratada. Cuadernos de Derecho Judicial, 7, 273-293.

Cabruja, T. (2004). Violencia doméstica: sexo y género en las teorías psicosociales sobre la violencia. Hacia otras propuestas de comprensión e intervención. Intervención Psicosocial, 3(2) 141154.

Consejería de Salud y Bienestar Social de Castilla y León (1991). Programa de intervención familiar en situaciones de desprotección infantil en Castilla y León. Castilla y León: Colección Acción Social.

Corral, P. (2000). Violencia contra la mujer. Debats, 70-71, 94-102.

Denuncias por malos tratos producidos por el cónyuge o análogo. Hombres y mujeres desde el 2000 al 2004. Instituto de la mujer. Obtenido el 19 de febrero de 2005 de htpp://www.mtas.es/ mujer/mcifras/VIOLENCI.HTM.

Departament de Benestar Social (2001). Serveis residencials d'estada limitada per a dones victimes de la violencia de gènere: cases d'acolliment i pisos amb suport. Documents de suport, 4, Barcelona: Generalitat de Catalunya.

Echeburua, E. (2004). Tratamiento del agresor doméstico. En J. Sanmartín (Ed.). El laberinto de la violencia. Causas, tipos y efectos. Barcelona: Ariel.

Echeburua, E., De Corral, P. Zubizarreta, I. y
Sarasua, B. (1996). Tratamiento cognitivo-conductual del trastorno de estrés postraumático en víctimas de maltrato doméstico: un estudio piloto. Análisis y modificación de Conducta, 22, 627654.

Fernández Villanueva, C. (2004). Violencia contra las mujeres: una visión estructural. Intervención Psicosocial, 13(2), 155-164.

Fontanil, Y., Ezama, E., Fernández, R., Gil, P., Herrero, F.J. y Paz, D. (2005). Prevalencia del maltrato de pareja contra las mujeres. Psicothema, 17, 90-95.

Giddens, A. (2000). La transformación de la intimidad. Sexualidad, amor y erotismo en las sociedades modernas. Madrid: Cátedra.

González, Fco. Estudio de los perfiles de familias de Servicios Sociales: Programas de Ayuda Social P.E.R. y P.E.P. del Ayuntamiento de Valencia" (Tesis Doctoral). Valencia, Universidad de Valencia, 2006.

Koop CE (1989). Violence against women. A global problem. Ginebra: Pan American Health Organization.

Neiding, P. y Friedman, C. (1988). Spouse abuse. A treatment program for couples. Illinois: Research Press Company.

Strube, M.J. y Barbour, L.S. (1984). Factors related to the decisión to leave an abusive relationship. Journal of Marriage and the Family, 46, 837-844.

Trigo, J. (1997). Indicadores de riesgo en familias atendidas por los servicios sociales. Apuntes de Psicología, 49-50, 153-170.

Trujano, P., Nava, C., Tejada, E. y Gutierrez, S. (2006). Estudio confirmatorio acerca de la frecuencia y percepción de la violencia. Intervención Psicosocial, 15(1), 95-110.

UNICEF (2000). Informe Innocenti. Obtenido el 2 de Septiembre de 2009 de http://www.uniceficdc.org/publications/pdf/digest6s.pdf.

Watts, C. Zimmerman, C. (2002). Violence against women: global scope magnitude. The Lancet, 359, 1232-1237.

Zacarés, A. (2005). La violencia de género explicada a mi hijo. Valencia: Carena editors.

Manuscrito recibido: 15/09/2009

Revisión recibida: 28/10/2009

Manuscrito aceptado: 29/10/2009 